\title{
Frequency Identification of Flexible Hub-Beam System Using Control Data
}

\author{
Xie Yong, Liu Pan and Cai Guo-Ping \\ Department of Engineering Mechanics, State Key Laboratory of Ocean Engineering, Shanghai Jiaotong Univer- \\ sity, Shanghai 200240, China
}

(Received 24 March 2014; accepted: 17 September 2014)

\begin{abstract}
This paper studies the parameter identification of a flexible hub-beam system based on the input-output data. Firstly, the first-order approximation coupling (FOAC) model is presented. Then, active position control for the system is studied using optimal tracking control theory. Finally, the observer/Kalman filter identification (OKID) and eigensystem realization algorithm (ERA) method are applied to identify the frequency of the system. In the simulations, the effectiveness of the identification method presented in this paper is verified by comparing the identification results of several different external excitations. Simulation results indicate that the anticipated position of the system may be traced by the proposed controller, and the residual vibration of the beam may be suppressed as well. The frequency of the system can be effectively identified using OKID and ERA. It is feasible and effective to identify the frequency using the control data.
\end{abstract}

\section{INTRODUCTION}

Spacecraft is composed of a central body and several flexible attachments. It is a typical rigid-flexible dynamic system with characteristics of dense frequencies and small damping. System assembly on the ground ( $1 \mathrm{G}$ gravity environment) is very difficult in many circumstances because of the flexibility of the structures; it is even more difficult to do vibration experiments on structures due to factors such as air damping, gravity effect, etc. Moreover, in some cases, the experimental apparatus may not be able to meet the experimental requirement of tests on the ground. On the other hand, flexible parameters of spacecraft, especially the natural frequencies of flexible solar array, may have great effect on the control of the spacecraft's attitude, since the flexible parameters will be used in control design. Due to the difference between ground and space environments, vibration behaviour of the solar array in two environments is different, too. So, the flexible parameters obtained by experiments on the ground cannot reflect the actual state of spacecraft in outer space. Therefore, it is necessary to study the on-orbit identification technology for the spacecraft to improve the accuracy in identifying flexible parameters. Since the flexible parameters obtained by the on-orbit identification are based on the real vibration of a spacecraft in outer space, it is more likely that these parameters are of high accuracy, and then control design based on these parameters can ensure high accuracy of attitude control. Furthermore, a system dynamic model may be modified by comparing the on-orbit test with the ground test so as to establish the quantitative relationships among the on-orbit test, the ground test, and the dynamic simulation. This will be helpful for the follow-up development of the spacecraft. The on-orbit identification needs the vibration response of spacecraft under certain excitation, which could be provided by the attitude manoeuvre, which means the realiza- tion of on-orbit identification is possible.

On-orbit parameter identification is conducted by directly using the input and output data, with no need of the exact dynamic model of the system. Generally speaking, the parameter identification methods can be divided into frequency domain methods and time domain methods, and the time domain method has better performance than the frequency domain method. Theoretical studies and engineering applications of the on-orbit identification technique have been completed in past research. For example, Haugse, et al. ${ }^{1}$ developed an accelerometer measurement system to gather data from an operational, on-orbit, and deployed space vehicle, and Fast Fourier Transform (FFT), Power Spectral Density (PSD) and Eigensystem Realization Algorithm (ERA) are employed for system modal identification. Kim, et al. ${ }^{2}$ presented a data processing strategy to generate equivalent free-decay responses from structural response data and used a free-decay time-domain modal identification technique for modal identification of Space Station Freedom. Tokio Kasai, et al. ${ }^{3}$ used the extended Kalman filter (EKF) technique to extract the modal parameters of a satellite with flexible solar panels. On the Engineering Test Satellite VI, impulse and random excitation were applied to the central body, and the measurement data were packed and downloaded via S-band digital serial telemetry for the off-line analysis by the ERA method. The identified modal parameters were then used in the synthesis of control law. ${ }^{4-8}$

On the International Space Station (ISS), the shuttle booster ignition pulse was used as an excitation to finish an on-orbit modal parameter identification test five times. The dynamic responses of the Shuttle-ISS mated structure were measured by the Shuttle payload bay video camera photogrammetric system, the Internal Wireless Instrumentation System (IWIS) ac- 


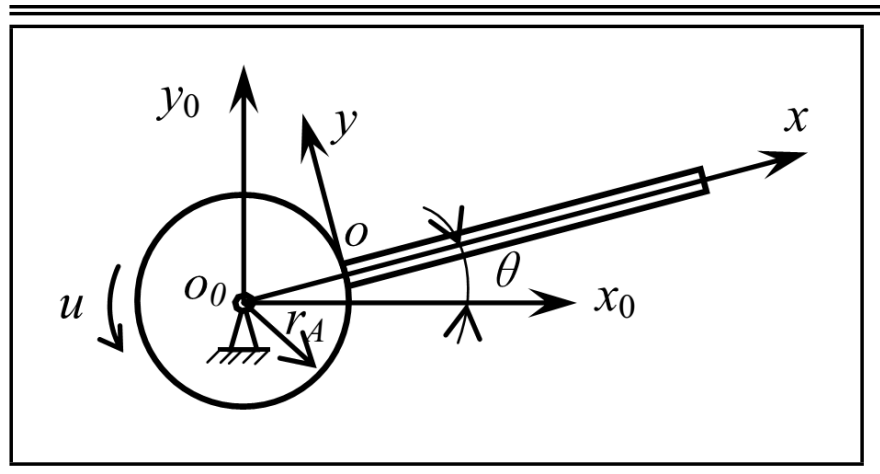

Figure 1. Structural model of the flexible hub-beam system.

celerometers, and the IWIS strain gauges. The measured data were processed and analysed based on the ERA to identify the structural modal parameters, including frequencies, damping, and mode shapes. ${ }^{9,10}$ On the Mir Space Station, the external excitations used in on-orbit modal experiment included Shuttle and Mir thruster firings, Shuttle-Mir and ProgressMir dockings crew exercise and push offs, and ambient noise during night-to-day and day-to-night orbital transitions. The data from the Mir Auxiliary Sensor Unit (MASU), which contains a total of 19 accelerometers, were used for modal identification by a time-domain free-decay method based on the ERA. ${ }^{11-14}$ It can be concluded from above that some practical work on spacecraft parameter identification has been done recently. However all the tests above are specially designed for parameter identification, which is separated from other space missions. Every space mission is extremely expensive, as is well known. We are then inspired to consider the following question: Can the attitude manoeuvre data of spacecraft be directly used for parameter identification? We need the dynamic response of spacecraft, which could be exactly provided by the attitude manoeuvre of spacecraft, to realize the on-orbit identification. If possible, it is unnecessary to carry out solely onorbit identification, saving costs on space missions. And it may also provide new methods for parameter identification in other fields.

In this paper, a frequency identification technique is investigated using a flexible hub-beam system as the object of the research, and the feasibility of identification using control data of the system is verified. The hub-beam system is a typical rigid-flexible coupling dynamic system. This system is applied to many engineering objects in practice, such as spacecraft, robots, turbine blades, etc. Today there are many studies on modelling and active control of the flexible hub-beam system. This paper is organized as follows: Section 2 briefly presents the first-order approximation coupling (FOAC) model for a flexible hub-beam system. Active controller design using optimal trajectory theory is presented in Section 3. Section 4 presents the OKID and ERA methods for the parameter identification. The numerical simulation results are shown in Section 5, and finally, Section 6 generalizes the conclusions of the research.

\section{THE FIRST-ORDER APPROXIMATION COUPLING MODEL}

A hub-beam rotating in the horizontal plane is considered here, as shown in Fig. 1, where the hub is a rigid body and the beam is flexible. The coordinate system $o_{0}-x_{0} y_{0}$ is an inertial frame, while the coordinate system $o-x y$ is fixed to the end of the beam, and this beam is fixed to the hub. The effect of gravity on the hub and the beam is neglected. The flexible beam is characterized by its length $L$, Young's modulus $E$, cross-sectional area moment of inertia $I$, mass density per unit volume $\rho$, and cross-sectional area $A$. The radius of the hub is $r_{A}, u$ is an external rotating torque acting on the hub, and $\theta$ describes the angular rotation of the hub. In the past few decades, researchers have proposed various dynamic models for the flexible hub-beam system, among which the zero-order approximation coupling (ZOAC) model and the first-order approximation coupling (FOAC) model are the most famous. The ZOAC model assumes the deformation in structural dynamics is small where axial and transverse displacements at any point in the beam are uncoupled; it may result in divergence to the system dynamic problem with high rotational speed, and it is only capable of solving cases with low rotational speed. In the FOAC model, the second-order coupling term of axial displacement caused by the transverse displacement of the flexible beam is considered, so the FOAC model is capable of both cases with low or high rotational speed. Cai, et al. conducted thorough studies on these two models. Details can be found in their research referenced in this study. ${ }^{15-18}$ In this paper, the FOAC model is adopted to describe the flexible hub-beam system, and is given below.

In many of the sources referenced here, ${ }^{15,16,18}$ the FOAC model of the hub-beam system is analysed in detail using the Hamilton's theory and the assumed-mode discretization method. It is expressed as

$$
\mathbf{M} \ddot{\mathbf{Y}}+\left(2 \dot{\theta} \mathbf{G}+\mathbf{C}_{t}\right) \dot{\mathbf{Y}}+\mathbf{K Y}=\mathbf{Q}+\mathbf{F}
$$

where

$$
\begin{aligned}
& \mathbf{Y}=\left[\begin{array}{l}
\theta \\
\mathbf{q}_{1} \\
\mathbf{q}_{2}
\end{array}\right], \quad \mathbf{M}=\left[\begin{array}{ccc}
J_{H}+M_{\theta \theta} & \mathbf{M}_{\theta q_{1}} & \mathbf{M}_{\theta q_{2}} \\
\mathbf{M}_{q_{1} \theta} & \mathbf{M}_{q_{1} q_{1}} & \mathbf{0} \\
\mathbf{M}_{q_{2} \theta} & \mathbf{0} & \mathbf{M}_{q_{2} q_{2}}
\end{array}\right], \\
& \mathbf{G}=\left[\begin{array}{lll}
0 & \mathbf{0} & \mathbf{0} \\
\mathbf{0} & \mathbf{0} & \mathbf{G}_{q_{1} q_{2}} \\
\mathbf{0} & \mathbf{G}_{q_{2} q_{1}} & \mathbf{0}
\end{array}\right], \quad \mathbf{Q}=\left[\begin{array}{c}
Q_{\theta} \\
\mathbf{Q}_{q_{1}} \\
\mathbf{0}
\end{array}\right], \quad \mathbf{F}=\left[\begin{array}{l}
u \\
\mathbf{0} \\
\mathbf{0}
\end{array}\right], \\
& \mathbf{K}=\left[\begin{array}{lll}
0 & \mathbf{0} & \mathbf{0} \\
\mathbf{0} & \mathbf{K}_{q_{1} q_{1}} & \mathbf{0} \\
\mathbf{0} & \mathbf{0} & \mathbf{K}_{q_{2} q_{2}}
\end{array}\right], \quad \mathbf{C}_{t}=\left[\begin{array}{ccc}
C_{H} & \mathbf{0} & \mathbf{0} \\
\mathbf{0} & \alpha_{1} \mathbf{M}_{1} & \mathbf{0} \\
\mathbf{0} & \mathbf{0} & \alpha_{2} \mathbf{M}_{2}
\end{array}\right]+ \\
& \frac{\beta_{1}}{\rho A}\left[\begin{array}{ccc}
M_{\theta \theta} & \mathbf{M}_{\theta q_{1}} & \mathbf{M}_{\theta q_{2}} \\
\mathbf{M}_{q_{1} \theta} & \mathbf{M}_{q_{1} q_{1}} & \mathbf{0} \\
\mathbf{M}_{q_{2} \theta} & \mathbf{0} & \mathbf{M}_{q_{2} q_{2}}
\end{array}\right]+\frac{\beta_{2} \dot{\theta} \operatorname{sgn}(\dot{\theta})}{\rho A}\left[\begin{array}{ccc}
C_{12} & \mathbf{C}_{21}^{T} & \mathbf{C}_{31}^{T} \\
\mathbf{C}_{21} & \mathbf{C}_{22} & \mathbf{0} \\
\mathbf{C}_{31} & \mathbf{0} & \mathbf{C}_{33}
\end{array}\right] ;
\end{aligned}
$$

and where $\mathbf{q}_{1}$ and $\mathbf{q}_{2}$ are $n \times 1$ vectors representing modal coordinates of axial and transverse vibrations of the beam, respectively. The parameter $M_{\theta \theta}$ is a scalar representing the 
total rotary inertia of the hub-beam system; $\mathbf{M}_{q_{1} q_{1}}=\mathbf{M}_{1}$ and $\mathbf{M}_{q_{2} q_{2}}=\mathbf{M}_{2}$, in which $\mathbf{M}_{1}$ and $\mathbf{M}_{2}$ are $n \times n$ generalized elastic mass matrices of the beam; $\mathbf{M}_{\theta q_{1}}=\mathbf{M}_{q_{1} \theta}^{T}$ and $\mathbf{M}_{\theta q_{2}}=\mathbf{M}_{q_{2} \theta}^{T}$ are $1 \times n$ vectors representing inertia effects caused by nonlinear coupling between large rotating motion and elastic deformation; $\mathbf{G}_{q_{1} q_{2}}$ and $\mathbf{G}_{q_{2} q_{1}}$ are $n \times n$ matrices resulted from gyroscopic effects; $C_{H}$ is the viscous damping coefficient of the bearing of hub; $\alpha_{1}$ and $\alpha_{2}$ are the damping coefficients of the beam in axial and transverse directions, respectively; $\beta_{1}$ and $\beta_{2}$ are the viscous and square damping coefficients caused by the windward side of the beam, respectively; $C_{11}$ is a scalar; $\mathbf{C}_{21}$ and $\mathbf{C}_{31}$ are both $n \times 1$ vectors; $\mathbf{C}_{22}$ and $\mathbf{C}_{33}$ are both $n \times n$ matrices; $\mathbf{K}_{q_{1} q_{1}}$ and $\mathbf{K}_{q_{2} q_{2}}$ are $n \times n$ stiffness matrices; $Q_{\theta}$ is a scalar; and $\mathbf{Q}_{q_{1}}$ is a $n \times 1$ vector. These quantities are defined as follows:

$$
\begin{aligned}
& M_{\theta \theta}=J_{1}+\mathbf{q}_{1}^{T} \mathbf{M}_{1} \mathbf{q}_{1}+\mathbf{q}_{2}^{T} \mathbf{M}_{2} \mathbf{q}_{2}+2\left(r_{A} \mathbf{U}_{01}+\mathbf{U}_{11}\right) \mathbf{q}_{1}- \\
& \mathbf{q}_{2}^{T}\left(r_{A} \mathbf{D}_{0}+\mathbf{D}_{1}\right) \mathbf{q}_{2} ; \\
& \mathbf{M}_{q_{1} \theta}=\mathbf{M}_{\theta q_{1}}^{T}=-\mathbf{R q}_{2} \\
& \mathbf{M}_{\theta q_{2}}=\mathbf{M}_{q_{2} \theta}^{T}=r_{A} \mathbf{U}_{02}+\mathbf{U}_{12}+\mathbf{q}_{1}^{T} \mathbf{R} ; \\
& \mathbf{M}_{q_{1} q_{1}}=\mathbf{M}_{1}=\int_{0}^{L} \rho A \boldsymbol{\Phi}_{1}^{T} \boldsymbol{\Phi}_{1} d x ; \\
& \mathbf{M}_{q_{2} q_{2}}=\mathbf{M}_{2}=\int_{0}^{L} \rho A \boldsymbol{\Phi}_{2}^{T} \boldsymbol{\Phi}_{2} d x \\
& \mathbf{G}_{q_{1} q_{2}}=-\mathbf{G}_{q_{2} q_{1}}^{T}=-\mathbf{R} \\
& \mathbf{K}_{q_{1} q_{1}}=\mathbf{K}_{1}-\dot{\theta}^{2} \mathbf{M}_{1} \\
& \mathbf{K}_{q_{2} q_{2}}=\mathbf{K}_{2}-\dot{\theta}^{2} \mathbf{M}_{2}+\dot{\theta}^{2}\left(r_{A} \mathbf{D}_{0}+\mathbf{D}_{1}\right) \text {; } \\
& Q_{\theta}=-2 \dot{\theta}\left[\left(\mathbf{q}_{1}^{T} \mathbf{M}_{1} \dot{\mathbf{q}}_{1}+\mathbf{q}_{2}^{T} \mathbf{M}_{2} \dot{\mathbf{q}}_{2}\right)+\left(r_{A} \mathbf{U}_{01}+\mathbf{U}_{11}\right) \dot{\mathbf{q}}_{1}-\right. \\
& \left.\mathbf{q}_{2}^{T}\left(r_{A} \mathbf{D}_{0}+\mathbf{D}_{1}\right) \dot{\mathbf{q}}_{2}\right] \\
& \mathbf{Q}_{q_{1}}=\dot{\theta}^{2}\left(r_{A} \mathbf{U}_{01}^{T}+\mathbf{U}_{11}^{T}\right) \text {; } \\
& C_{11}=\int_{0}^{L} \rho A\left(r_{A}+x\right)^{3} d x+\mathbf{q}_{1}^{T}\left[r_{A} \mathbf{M}_{1}+\right. \\
& \left.\int_{0}^{L} \rho A x \boldsymbol{\Phi}_{1}^{T} \boldsymbol{\Phi}_{1} d x\right] \mathbf{q}_{1}+\mathbf{q}_{2}^{T}\left[r_{A} \mathbf{M}_{2}+\int_{0}^{L} \rho A x \boldsymbol{\Phi}_{2}^{T} \boldsymbol{\Phi}_{2} d x\right] \mathbf{q}_{2}+ \\
& 2\left[r_{A}^{2} \mathbf{U}_{01}+2 r_{A} \mathbf{U}_{11}+\int_{0}^{L} \rho A x^{2} \boldsymbol{\Phi}_{1} d x\right] \mathbf{q}_{1}- \\
& \mathbf{q}_{2}^{T}\left[r_{A}^{2} \mathbf{D}_{0}+2 r_{A} \mathbf{D}_{1}+\int_{0}^{L} \rho A x^{2} \mathbf{S}(x) d x\right] \mathbf{q}_{2} ; \\
& \mathbf{C}_{21}=-\left[r_{A} \mathbf{R}+\int_{0}^{L} \rho A x \boldsymbol{\Phi}_{1}^{T} \boldsymbol{\Phi}_{2} d x\right] \mathbf{q}_{2} ; \\
& \mathbf{C}_{31}=r_{A}^{2} \mathbf{U}_{02}^{T}+2 r_{A} \mathbf{U}_{12}^{T}+\int_{0}^{L} \rho A x^{2} \boldsymbol{\Phi}_{2}^{T} d x+ \\
& {\left[r_{A} \mathbf{R}+\int_{0}^{L} \rho A x \boldsymbol{\Phi}_{2}^{T} \boldsymbol{\Phi}_{1} d x\right] \mathbf{q}_{1}} \\
& \mathbf{C}_{22}=r_{A} \mathbf{M}_{1}+\int_{0}^{L} \rho A x \boldsymbol{\Phi}_{1}^{T} \boldsymbol{\Phi}_{1} d x ; \\
& \mathbf{C}_{33}=r_{A} \mathbf{M}_{2}+\int_{0}^{L} \rho A x \boldsymbol{\Phi}_{2}^{T} \boldsymbol{\Phi}_{2} d x ;
\end{aligned}
$$

where $\mathbf{K}_{1}$ and $\mathbf{K}_{2}$ in Eqs. (8) and (9) are $n \times n$ generalized elastic stiffness matrices of the beam. Parameters $\boldsymbol{\Phi}_{1}(x)$ and
$\boldsymbol{\Phi}_{2}(x)$ in the above equations are $1 \times n$ vectors. They represent mode functions of axial and transverse vibrations of the beam, respectively, and they assume mode functions of the cantilever beam. The constant parameters in Eqs. (2)-(11) are given as follows:

$$
\begin{aligned}
J_{1} & =\int_{0}^{L} \rho A\left(r_{A}+x\right)^{2} d x ; \\
\mathbf{K}_{1} & =\int_{0}^{L} E A \boldsymbol{\Phi}_{1}^{\prime T} \boldsymbol{\Phi}_{1}^{\prime} d x ; \\
\mathbf{K}_{2} & =\int_{0}^{L} E I \boldsymbol{\Phi}_{2}^{\prime \prime T} \boldsymbol{\Phi}_{2}^{\prime \prime} d x ; \\
\mathbf{U}_{0 j} & =\int_{0}^{L} \rho A \boldsymbol{\Phi}_{j} d x, \quad j=1,2 ; \\
\mathbf{U}_{1 j} & =\int_{0}^{L} \rho A x \boldsymbol{\Phi}_{j} d x, \quad j=1,2 ; \\
\mathbf{D}_{0} & =\int_{0}^{L} \rho A \mathbf{S}(x) d x ; \\
\mathbf{D}_{1} & =\int_{0}^{L} \rho A x \mathbf{S}(x) d x ; \\
& \mathbf{R}=\int_{0}^{L} \rho A \boldsymbol{\Phi}_{1}^{T} \boldsymbol{\Phi}_{2} d x ;
\end{aligned}
$$

where $J_{1}$ is a scalar; $\mathbf{U}_{0 j}$ and $\mathbf{U}_{1 j}$ are both $1 \times n$ vectors; and $\mathbf{D}_{0}, \mathbf{D}_{1}$, and $\mathbf{R}$ are all $n \times n$ matrices. The parameter $\mathbf{S}(x)$ in Eqs. (22) and (23) is the coupling shape function, and this $n \times n$ matrix, discussed in sources used here, ${ }^{15,16,18}$ is given by

$$
\mathbf{S}(x)=\int_{0}^{x} \boldsymbol{\Phi}_{2}^{\prime T}(\xi) \boldsymbol{\Phi}_{2}^{\prime}(\xi) d \xi
$$

\section{ACTIVE CONTROL DESIGN}

The FOAC model given above is nonlinear and timevarying. Control design directly using this model is difficult, since nonlinear control theory is still under intensive and continuous development, though nowadays linear control theory is comprehensive, and linear control design has many practical applications. Here, we linearise the FOAC model, and use a linear optimal tracking control method to design a controller to control the flexible hub-beam system.

\subsection{Linearisation of the FOAC Model}

The effect of axial vibrations of the flexible beam on system dynamics is much smaller than that of transverse vibrations, and can be neglected. In classical linearisation for the flexible hub-beam system, it is often assumed that the angular velocity of rotational motion is small, so the related terms and their higher order terms are neglected. ${ }^{19}$ Increment of rotary inertia of the beam caused by its elastic deformation is also small, which results in the omission of terms related to $\mathbf{q}_{1}$ and $\mathbf{q}_{2}$ in Eq. (2). ${ }^{19}$ So, the linearised model of hub-beam system can be 
written as ${ }^{18,19}$

$$
\begin{gathered}
{\left[\begin{array}{cc}
J_{H}+J_{1} & \mathbf{M}_{\theta q_{2}} \\
\mathbf{M}_{q_{2} \theta} & \mathbf{M}_{2}
\end{array}\right]\left[\begin{array}{c}
\ddot{\theta} \\
\ddot{\mathbf{q}}_{2}
\end{array}\right]+\left[\begin{array}{cc}
C_{H}+\frac{\beta_{1}}{\rho A} J_{1} & \frac{\beta_{1}}{\rho A} \mathbf{M}_{\theta q_{2}} \\
\frac{\beta_{1}}{\rho A} \mathbf{M}_{q_{2} \theta} & \left(\alpha_{2}+\frac{\beta_{1}}{\rho A}\right) \mathbf{M}_{2}
\end{array}\right]\left[\begin{array}{c}
\dot{\theta} \\
\dot{\mathbf{q}}_{2}
\end{array}\right]+} \\
{\left[\begin{array}{cc}
0 & \mathbf{0} \\
\mathbf{0} & \mathbf{K}_{2}
\end{array}\right]\left[\begin{array}{c}
\theta \\
\mathbf{q}_{2}
\end{array}\right]=\left[\begin{array}{l}
u \\
\mathbf{0}
\end{array}\right]}
\end{gathered}
$$

where $J_{1}, \mathbf{M}_{\theta q_{2}}, \mathbf{M}_{q_{2}}, \mathbf{M}_{2}$, and $\mathbf{K}_{2}$ are given by Eqs. (17), (4), (6) and (19), respectively. In the expressions of $\mathbf{M}_{\theta q_{2}}$ and $\mathbf{M}_{q_{2} \theta}$, the term of $\mathbf{q}_{1}^{T} \mathbf{R}$ should be neglected.

In matrix form, Eq. (26) may be written as

$$
\hat{\mathbf{M}} \ddot{\hat{\mathbf{Y}}}+\hat{\mathbf{C}} \dot{\hat{\mathbf{Y}}}+\hat{\mathbf{K}} \hat{\mathbf{Y}}=\overline{\mathbf{H}} u
$$

where $\hat{\mathbf{Y}}=\left[\begin{array}{c}\theta \\ \mathbf{q}_{2}\end{array}\right], \hat{\mathbf{M}}=\left[\begin{array}{cc}J_{H}+J_{B} & \mathbf{M}_{\theta q_{2}} \\ \mathbf{M}_{q_{2} \theta} & \mathbf{M}_{2}\end{array}\right], \hat{\mathbf{C}}=$ $\left[\begin{array}{cc}C_{H}+\frac{\beta_{1}}{\rho A} J_{1} & \frac{\beta_{1}}{\rho A} \mathbf{M}_{\theta q_{2}} \\ \frac{\beta_{1}}{\rho A} \mathbf{M}_{q_{2} \theta} & \left(\alpha_{2}+\frac{\beta_{1}}{\rho A}\right) \mathbf{M}_{2}\end{array}\right], \hat{\mathbf{K}}=\left[\begin{array}{cc}0 & \mathbf{0} \\ \mathbf{0} & \mathbf{K}_{2}\end{array}\right]$, and $\overline{\mathbf{H}}=\left[\begin{array}{l}1 \\ \mathbf{0}\end{array}\right]$. In state-space formulation, Eq. (27) becomes

$$
\dot{\mathbf{x}}=\overline{\mathbf{A}} \mathbf{x}+\overline{\mathbf{B}} u
$$

$$
\begin{aligned}
& \text { where } \mathbf{x}=\left[\begin{array}{c}
\hat{\mathbf{Y}} \\
\dot{\hat{\mathbf{Y}}}
\end{array}\right], \overline{\mathbf{A}}=\left[\begin{array}{cc}
\mathbf{0} & \mathbf{I}_{2(n+1) \times 2(n+1)} \\
-\hat{\mathbf{M}}^{-1} \hat{\mathbf{K}} & -\hat{\mathbf{M}}^{-1} \hat{\mathbf{C}}
\end{array}\right] \text {, and } \\
& \overline{\mathbf{B}}=\left[\begin{array}{c}
\mathbf{0} \\
\hat{\mathbf{M}}^{-1} \overline{\mathbf{H}}
\end{array}\right] \text {. }
\end{aligned}
$$

\subsection{Controller Design}

The optimal tracking controller is investigated in this section. The desired state trajectory of the system is assumed to be given by $\mathbf{x}_{d}=\left[\hat{\mathbf{Y}}_{d}^{T}, \dot{\hat{\mathbf{Y}}}_{d}^{T}\right]^{T}$, where $\hat{\mathbf{Y}}_{d}^{T}=\left[\theta_{d}, \mathbf{q}_{2 d}^{T}\right]^{T}$. The following state error vector is defined as

$$
\mathbf{e}=\mathbf{x}_{d}-\mathbf{x}
$$

and the performance index is defined as

$$
J=\frac{1}{2} \int_{0}^{\infty}\left(\mathbf{e}^{T} \mathbf{Q} \mathbf{e}+\bar{R} u^{2}\right) d t
$$

where $\mathbf{Q}$ is a $2(n+1) \times 2(n+1)$ weighting matrix, which is positive definite and symmetric, and $\bar{R}$ is a positive weighting scalar. The optimal tracking control law may be obtained as ${ }^{20}$

$$
u=-\bar{R}^{-1} \overline{\mathbf{B}}^{T} \mathbf{P} \mathbf{x}+\bar{R}^{-1} \overline{\mathbf{B}}^{T}\left[\mathbf{P} \overline{\mathbf{B} R}^{-1} \overline{\mathbf{B}}^{T}-\overline{\mathbf{A}}^{T}\right]^{-1} \mathbf{Q} \mathbf{x}_{d}
$$

where $\mathbf{P}$ is the solution of the Riccati equation

$$
\mathbf{P} \overline{\mathbf{A}}+\overline{\mathbf{A}}^{T} \mathbf{P}-\mathbf{P} \overline{\mathbf{B} R}^{-1} \overline{\mathbf{B}}^{T} \mathbf{P}=-\mathbf{Q} .
$$

In the controller Eq. (31), the first term is a linear function of state $\mathbf{x}$, representing the regulating role of negative feedback. The second term is a linear function of the desired state $\mathbf{x}_{d}$, representing a driving action resulting from $\mathbf{x}_{d}$.

\section{PARAMETER IDENTIFICATION OF THE SYSTEM}

In this section, parameter identification technology will be investigated for the system. The observer/Kalman filter identification (OKID) and eigensystem realization algorithm (ERA) will be used in the parameter identification. These two methods are both time-domain identification techniques and have successful applications in spacecraft.

\subsection{Description for OKID Technique}

The OKID is a time-domain identification technique, proposed by Juang, et al. ${ }^{21}$ Consider the following discrete linear system

$$
\left\{\begin{array}{l}
\mathbf{x}(k+1)=\mathbf{A} \mathbf{x}(k)+\mathbf{B} u(k) \\
\mathbf{y}(k)=\mathbf{C x}(k)+\mathbf{D} u(k)
\end{array} ;\right.
$$

where $\mathbf{x}$ is $2(n+1)$-dimensional state vector; $u$ is the input torque; and $\mathbf{y}$ is $q \times 1$ output vector. The matrices $\mathbf{A}, \mathbf{B}, \mathbf{C}$, and $\mathbf{D}$ are the system matrix, input matrix, output matrix, and influence matrix, respectively. From the recursive relations of Eq. (33), one can obtain the expression of the system output at $k \bar{T}$ moment ( $\bar{T}$ denotes the data sampling period) as

$$
\begin{aligned}
\mathbf{y}(k) & =\mathbf{C A}^{k} \mathbf{x}(0)+\sum_{\tau=0}^{k-1} \mathbf{C A}^{\tau} \mathbf{B} u(k-\tau-1)+\mathbf{D} u(k) \\
& =\mathbf{C A}^{k} \mathbf{x}(0)+\sum_{\tau=0}^{k-1} \mathbf{Y}_{\tau} u(k-\tau-1)+\mathbf{D} u(k) ;
\end{aligned}
$$

where $\mathbf{Y}_{\tau}=\mathbf{C A}^{\tau} \mathbf{B}$ and $\mathbf{D}$ are the system Markov parameters to be identified. If the initial condition of the system is zero or given, i.e., if $\mathbf{x}(0)$ at $k=0$ is known, the system Markov parameters can be determined directly using Eq. (34). ${ }^{21}$ However, for a practical engineering structure, the exact value of initial condition is difficult to determine. Even though the structure is at an equilibrium state and does not suffer from any external excitation, the zero initial condition cannot be exactly guaranteed; yet, there are always certain affecting factors that make it impossible for the initial condition to be zero. Errors may occur inevitably when the system Markov parameters are identified using Eq. (34) based on the input-output data of the system. To eliminate the influence of the initial conditions on the identification of system Markov parameters, we firstly construct a state observer whose Markov parameter equation is independent of the initial condition, and by solving this equation based on the input-output data to get the observer's parameters, and then by establishing the relationship of Markov parameters between the observer and the original system, we finally work out the Markov parameters of the original system. The detailed process is described below.

The following state observer is constructed as ${ }^{21}$

$$
\left\{\begin{array}{rl}
\widehat{\mathbf{x}}(k+1) & =\mathbf{A} \widehat{\mathbf{x}}(k)+\mathbf{B} u(k)-\mathbf{F}[\mathbf{y}(k)-\widehat{\mathbf{y}}(k)] \\
& =(\mathbf{A}+\mathbf{F C}) \widehat{\mathbf{x}}(k)+(\mathbf{B}+\mathbf{F D}) u(k)-\mathbf{F y}(k) ; \\
\widehat{\mathbf{y}}(k)=\mathbf{C} \widehat{\mathbf{x}}(k)+\mathbf{D} u(k)
\end{array} ;\right.
$$


where $\mathbf{F}$ is the weighting matrix of the observer. The eigenvalues of $\mathbf{A}+\mathbf{F C}$ are adjusted by the selection of $\mathbf{F}$ such that the state $\widehat{\mathbf{x}}(k)$ of the observer approaches the real state $\mathbf{x}(k)$ of the system. The output of the observer at $k \bar{T}$ moment can be written as

$$
\begin{aligned}
& \widehat{\mathbf{y}}(k)=\mathbf{C}(\mathbf{A}+\mathbf{F C})^{k} \widehat{\mathbf{x}}(0)+ \\
& \quad \sum_{\tau=0}^{k-1} \widehat{\mathbf{Y}}_{\tau}\left[u^{T}(k-\tau-1) \mathbf{y}^{T}(k-\tau-1)\right]^{T}+\mathbf{D} u(k) ;
\end{aligned}
$$

where $\widehat{\mathbf{Y}}_{\tau}$ and $\mathbf{D}$ are the observer Markov parameters; $\widehat{\mathbf{Y}}_{\tau}$ is expressed as

$$
\begin{aligned}
\widehat{\mathbf{Y}}_{\tau} & =\left[\begin{array}{ll}
\mathbf{C}(\mathbf{A}+\mathbf{F C})^{\tau}(\mathbf{B}+\mathbf{F D}) & -\mathbf{C}(\mathbf{A}+\mathbf{F C})^{\tau} \mathbf{F}
\end{array}\right] \\
& =\left[\begin{array}{ll}
\widehat{\mathbf{Y}}_{\tau}^{(1)} & \widehat{\mathbf{Y}}_{\tau}^{(2)}
\end{array}\right]
\end{aligned}
$$

where $\widehat{\mathbf{Y}}_{\tau}^{(1)}=\mathbf{C}(\mathbf{A}+\mathbf{F C})^{\tau}(\mathbf{B}+\mathbf{F D})$ and $\widehat{\mathbf{Y}}_{\tau}^{(2)}=-\mathbf{C}(\mathbf{A}+$ $\mathbf{F C})^{\tau} \mathbf{F}$.

By selecting $\mathbf{F}$ to make the pole of $\mathbf{A}+\mathbf{F C}$ on the origin, the eigenvalue equation of $\mathbf{A}+\mathbf{F C}$ will be $\left(\lambda-\lambda_{i}\right)^{2(n+1)}$; where $\lambda_{i}$ is the $i$-th eigenvalue. Then by the Hamilton-Cayley theorem, we have $(\mathbf{A}+\mathbf{F C})^{2(n+1)}=\mathbf{0}$. From Eqs. (33) and (34) and by some derivation, the state error equation can be written as

$$
\mathbf{x}(k+1)-\widehat{\mathbf{x}}(k+1)=(\mathbf{A}+\mathbf{F C})[\mathbf{x}(k)-\widehat{\mathbf{x}}(k)] .
$$

By the recurrence of the state error equation, when $k \geq n$, we have

$$
\begin{aligned}
\mathbf{x}(k+1)- & \widehat{\mathbf{x}}(k+1)=(\mathbf{A}+\mathbf{F C})[\mathbf{x}(k)-\widehat{\mathbf{x}}(k)] \\
= & (\mathbf{A}+\mathbf{F C})^{2}[\mathbf{x}(k-1)-\widehat{\mathbf{x}}(k-1)] \\
& \vdots \\
= & (\mathbf{A}+\mathbf{F C})^{2(n+1)}[\mathbf{x}(k-2 n-1)-\widehat{\mathbf{x}}(k-2 n-1)] \\
= & \mathbf{0}
\end{aligned}
$$

From Eq. (39), when $k \geq 2(n+1)$, the state $\widehat{\mathbf{x}}(k)$ of the observer will converge to the real state $\mathbf{x}(k)$, i.e., $\mathbf{x}(k)=\widehat{\mathbf{x}}(k)$ $(k \geq 2(n+1))$. So, from the second equation of Eq. (35), the output $\widehat{\mathbf{y}}(k)$ of the observer will converge to the real output $\mathbf{y}(k)$ when $k \geq 2(n+1)$, i.e., $\mathbf{y}(k)=\widehat{\mathbf{y}}(k)(k \geq 2(n+1))$. From Eq. (37), we know that $\widehat{\mathbf{Y}}_{\tau}=\mathbf{0}$ when $\tau \geq 2(n+1)$. Therefore, when $k \geq 2(n+1)$, Eq. (36) can be written as

$$
\begin{gathered}
\mathbf{y}(k)=\sum_{\tau=0}^{2 n+1} \widehat{\mathbf{Y}}_{\tau}\left[u(k-\tau-1) \mathbf{y}^{T}(k-\tau-1)\right]^{T}+\mathbf{D} u(k) \\
k \geq 2(n+1)
\end{gathered}
$$

Equation (40) holds strictly and is independent of the initial condition of the system. But Eq. (36) is subject to the initial condition. Using the least squares to solve Eq. (40), the Markov parameters $\widehat{\mathbf{Y}}_{\tau}$ and $\mathbf{D}$ of the observer can be obtained. ${ }^{21}$ The relationship of Markov parameters between the original system and the observer is ${ }^{21,22}$

$$
\mathbf{Y}_{\tau}=\mathbf{C A}^{\tau} \mathbf{B}=\widehat{\mathbf{Y}}_{\tau}^{(1)}+\sum_{k=0}^{\tau-1} \widehat{\mathbf{Y}}_{k}^{(2)} \widehat{\mathbf{Y}}_{\tau-k-1}+\widehat{\mathbf{Y}}_{\tau}^{(2)} \mathbf{D}
$$

Based on the input-output data of the system, Markov parameters of the observer can be obtained from Eq. (40), and Markov parameters of the original system can be obtained from Eq. (41). The minimal state-space realization of the system can be determined by using the eigensystem realization algorithm (ERA) given in the following Section 4.2. From the above process, we can observe that the specific value of $\mathbf{F}$ is not needed in the entire calculation for Markov parameters.

\subsection{Eigensystem Realization Algorithm (ERA)}

The ERA is a well-established algorithm for parameter identification in time domain. It utilizes impulse response data of the system to seek the minimal state-space realization $\left(\mathbf{A}_{r}, \mathbf{B}_{r}, \mathbf{C}_{r}, \mathbf{D}_{r}\right)$ by the singular value decomposition of the Hankel block matrix.

The $(\bar{s}+1) \times(\bar{t}+1)$ Hankel block matrix is constructed $\operatorname{as}^{23,24}$

$$
\mathbf{H}(\tau)=\left[\begin{array}{cccc}
\mathbf{Y}_{\tau} & \mathbf{Y}_{\tau+1} & \cdots & \mathbf{Y}_{\tau+\bar{t}} \\
\mathbf{Y}_{\tau+1} & \mathbf{Y}_{\tau+2} & \cdots & \mathbf{Y}_{\tau+1+\bar{t}} \\
\vdots & \vdots & \ddots & \vdots \\
\mathbf{Y}_{\tau+\bar{s}} & \mathbf{Y}_{\tau+\bar{s}+1} & \cdots & \mathbf{Y}_{\tau+\bar{s}+\bar{t}}
\end{array}\right]
$$

The matrix $\mathbf{H}(\tau)$ can be written as the following form:

$$
\mathbf{H}(\tau)=\overline{\mathbf{V}}_{\bar{s}} \mathbf{A}^{\tau} \overline{\mathbf{W}}_{\bar{t}}
$$

where $\overline{\mathbf{V}}_{\bar{s}}=\left[\mathbf{C}^{T}(\mathbf{C A})^{T}\left(\mathbf{C A}^{2}\right)^{T} \ldots\left(\mathbf{C A}^{\bar{s}}\right)^{T}\right]^{T}, \overline{\mathbf{W}}_{\bar{t}}=$ $\left[\begin{array}{lllll}\mathbf{B} & \mathbf{A B} & \mathbf{A}^{2} \mathbf{B} & \ldots & \mathbf{A}^{\bar{t}} \mathbf{B}\end{array}\right]$ are the controllable and observable matrices of the system, respectively. It is well known that for the system with the order $2(n+1)$, the minimal dimension of the system matrix is $2(n+1) \times 2(n+1)$. If $\bar{s}+1 \geq 2(n+1)$, $\bar{t}+1 \geq 2(n+1)$, and the system is controllable and observable, the rank of $\overline{\mathbf{V}}_{\bar{s}}$ and $\overline{\mathbf{W}}_{\bar{t}}$ will be both $n$; therefore, the rank of the Hankel block matrix $\mathbf{H}(\tau)$ will be $2(n+1)$. By performing the singular value decomposition of $\mathbf{H}(0)$, we have

$$
\mathbf{H}(0)=\mathbf{U N V}^{T} ;
$$

where $\mathbf{U}$ and $\mathbf{V}$ are both unitary matrix, and $\mathbf{N}$ is the eigenvalue diagonal matrix, given by

$$
\begin{gathered}
\mathbf{N}=\operatorname{diag}\left(d_{1}, d_{2}, \ldots, d_{r}, d_{r+1}, \ldots\right), \\
d_{1} \geq d_{2} \geq \ldots \geq d_{r} \geq d_{r+1} \geq \ldots \geq 0 .
\end{gathered}
$$

Assuming that the order of minimal realization of the system is $r, r$ can be determined using the following singular value truncation threshold $\varepsilon$ :

$$
\frac{d_{r}}{d_{1}} \geq \varepsilon, \quad \frac{d_{r+1}}{d_{1}} \leq \varepsilon .
$$

Let $\mathbf{U}_{r}$ be the first $r$ columns of $\mathbf{U}$, let $\mathbf{V}_{r}$ be the first $r$ columns of $\mathbf{V}$, and let $\mathbf{N}_{r}=\operatorname{diag}\left(d_{1}, d_{2}, \ldots, d_{r}\right)$. Now define the following matrices:

$$
\mathbf{E}_{q}^{T}=\left[\begin{array}{ll}
\mathbf{I}_{q} & \mathbf{0}_{q \times(l-1) q}
\end{array}\right], \quad \mathbf{E}_{m}^{T}=\left[\begin{array}{ll}
\mathbf{I}_{m} & \mathbf{0}_{m \times(l-1) m}
\end{array}\right] ;
$$


where $\mathbf{I}_{q}$ and $\mathbf{I}_{m}$ are $q$-dimensional and $m$-dimensional unit matrices, respectively. The minimal realization $\left(\mathbf{A}_{r}, \mathbf{B}_{r}, \mathbf{C}_{r}, \mathbf{D}_{r}\right)$ of the system can be determined as ${ }^{23,24}$

$$
\begin{aligned}
& \mathbf{A}_{r}=\mathbf{N}_{r}^{-1 / 2} \mathbf{U}_{r}^{T} \mathbf{H}(1) \mathbf{V}_{r} \mathbf{N}_{r}^{-1 / 2}, \\
& \mathbf{B}_{r}=\mathbf{N}_{r}^{1 / 2} \mathbf{V}_{r} \mathbf{E}_{m}, \\
& \mathbf{C}_{r}=\mathbf{E}_{q}^{T} \mathbf{U}_{r} \mathbf{N}_{r}^{1 / 2} .
\end{aligned}
$$

By solving the eigenvalues of $\mathbf{A}_{r}$, we can obtain

$$
\boldsymbol{\psi}^{-1} \mathbf{A}_{r} \boldsymbol{\psi}=\boldsymbol{\Lambda}, \quad \boldsymbol{\Lambda}=\operatorname{diag}\left(\bar{\lambda}_{1}, \bar{\lambda}_{2}, \ldots, \bar{\lambda}_{2(n+1)}\right)
$$

where $\boldsymbol{\psi}$ is the eigenvector matrix of $\mathbf{A}_{r}$ and $\bar{\lambda}_{i}(i=$ $1,2, \ldots, 2(n+1))$ is the $i$-th eigenvalue. Define the following parameter:

$$
s_{i}=\frac{\ln \left(\bar{\lambda}_{i}\right)}{T}, \quad i=1,2, \ldots, 2(n+1) ;
$$

where $T$ is the data sampling period. Then, the natural frequency $\omega_{i}$ can be obtained as

$$
\omega_{i}=\sqrt{\left[\operatorname{Re}\left(s_{i}\right)\right]^{2}+\left[\operatorname{Im}\left(s_{i}\right)\right]^{2}}, \quad i=1,2, \ldots, 2(n+1) ;
$$

where $\operatorname{Re}\left(s_{i}\right)$ and $\operatorname{Im}\left(s_{i}\right)$ are the real part and imaginary part of $s_{i}$, respectively.

\section{NUMERICAL SIMULATIONS}

In this section, numerical simulations are carried out to demonstrate the effectiveness of the identification method proposed in this paper. The radius of the hub is assumed to be $r_{A}=0.05 \mathrm{~m}$, and its rotary inertia is $J_{H}=0.30 \mathrm{kgm}^{2}$. The properties of the flexible beam are given as follows: The length is $L=1.8 \mathrm{~m}$, the cross-section area is $A=2.5 \times 10^{-4} \mathrm{~m}^{2}$, the area rotary inertia is $I=1.3021 \times 10^{-10} \mathrm{~m}^{4}$, the mass density is $\rho=2.766 \times 10^{3} \mathrm{~kg} / \mathrm{m}^{3}$, and the modulus of elasticity is $E=6.90 \times 10^{10} \mathrm{~N} / \mathrm{m}^{2}$. The damping parameters adopted in Yang's study of dynamic modelling theory ${ }^{25}$ are set to $\alpha_{1}=\alpha_{2}=0.011, \beta_{1}=0, \beta_{2}=0.0353$, and $C_{H}=0$. For the flexible cantilever beam with no large motion, the fundamental frequency is $0.622 \mathrm{~Hz}$.

Assume that the following rotating torque is put on the hub:

$$
u(t)= \begin{cases}u_{0} \sin \left(\frac{2 \pi}{T} t\right), & 0 \leq t \leq T \\ 0 & t>T\end{cases}
$$

where $T=2 \mathrm{~s}$ and $u_{0}=1 \mathrm{Nm}$. The tip transverse response of the beam and the time history of angle displacement of large motion of the hub are displayed in Fig. 2. The domain frequency of the tip transverse response of the beam based on the FOAC model is the first-order frequency $(1.381 \mathrm{~Hz})$, and the weightiness of the second order frequency is very small. Due to the effect of large motion, the first-order frequency now is 2.2 times that of the fundamental frequency without rotation. This is the so-called dynamic stiffening phenomenon, which results from the additional stiffness caused by the coupling of
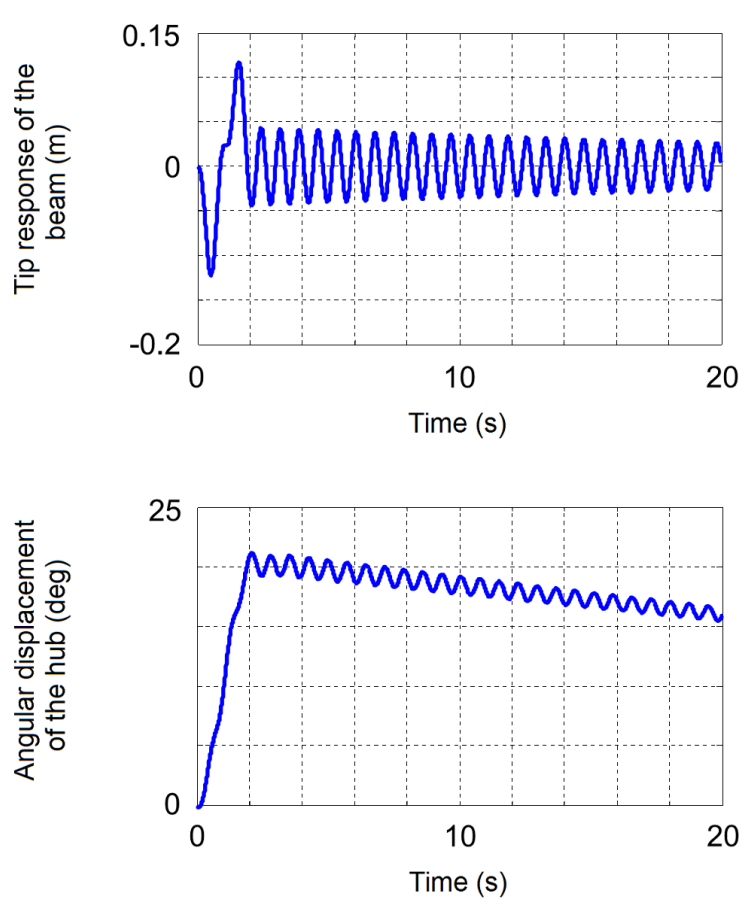

Figure 2. The tip response of the beam and the time history of angular displacement of the hub.

large rotation of the rigid hub and small elastic vibration of the flexible beam. ${ }^{25}$ In Yang's study, dynamics experiments of flexible hub-beam system were done, in which several beams of different lengths were taken into account, and the experimental results indicate that the domain vibration frequency of the beam with rotation is always about 2.2 times that of the fundamental frequency of the beam without rotation. ${ }^{25}$ Based on this conclusion, the first-order frequency is set to be the target of parameter identification.

In the identification simulations, three cases are considered: white-noise excitation, position control, and constant-speed rotation control. A white-noise case means that the external torque on the hub is a white-noise signal. A position control case means that a controller on the hub is designed to drive the system from one position to another, and the residual vibration of the beam should be suppressed by this controller after the arrival of position. Constant-speed rotation control means that a controller is designed to drive the system to rotate with a constant angular velocity, and the residual vibration of the beam should be suppressed. The angular displacement and angular velocity of the hub, and the tip transverse response of the beam, will be used as the output of the system in the parameter identifications. The detailed input-output arrangements are:

- Case 1: white-noise torque is used as the input of the system; the angular velocity of the hub and the tip transverse response of the beam are used as the output of the system, respectively.

- Case 2: the position controller is used as the input; the angular displacement of the hub and the tip transverse re- 


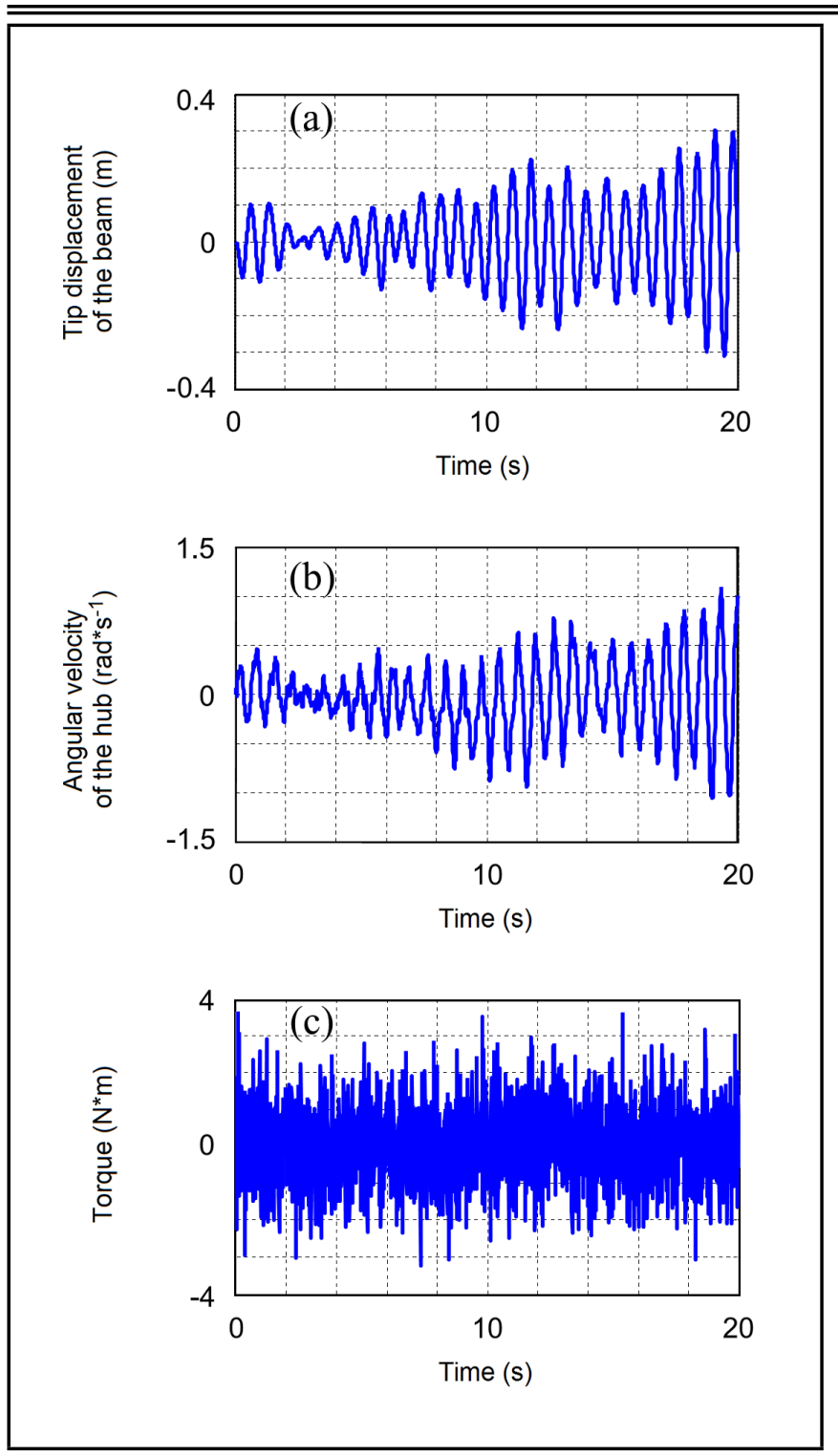

Figure 3. The responses of the system under white-noise excitation: (a) tip displacement of the beam, (b) angular velocity of the hub, and (c) white-noise excitation.

sponse of the beam are used as the output, respectively.

- Case 3: the constant-speed rotation controller is used as the input; the angular velocity of the hub and the tip transverse response of the beam are used as the output, respectively.

Then, parameter identifications will be done for the above three cases. The data sampling period is all chosen as $100 \mathrm{~Hz}$ in the simulations. First, we consider the white-noise case. The tip transverse response of the beam, the time history of angular velocity of the hub, and white noise torque are displayed in Fig. 3. So, the input and output data of the system are both known; thus the OKID and ERA may be used to identify the first-order frequency of the system, and the results are given in the third and fourth rows of Table 1. The theoretical result is given in the second row of Table 1 for comparison, as well. We can observe from Table 1 that the identification results are very close to the theoretical ones, which proves the validity of the methods in this paper.
Table 1. The identification results of the first-order frequency of the system.

\begin{tabular}{|c|c|c|c|}
\hline Case & Output signal & Frequency $(\mathrm{Hz})$ & Error (\%) \\
\hline $\begin{array}{l}\text { Theoretical } \\
\text { results }\end{array}$ & & 1.3810 & \\
\hline \multirow{2}{*}{$\begin{array}{l}\text { White-noise } \\
\text { excitation }\end{array}$} & $\begin{array}{l}\text { Tip transverse } \\
\text { response of beam }\end{array}$ & 1.3841 & 0.224475 \\
\hline & $\begin{array}{l}\text { Angular velocity } \\
\text { of beam }\end{array}$ & 1.3834 & 0.173787 \\
\hline \multirow{2}{*}{$\begin{array}{l}\text { Position } \\
\text { control }\end{array}$} & $\begin{array}{l}\text { Tip transverse } \\
\text { response of beam }\end{array}$ & 1.3762 & 0.347574 \\
\hline & $\begin{array}{l}\text { Angular displace- } \\
\text { ment of hub }\end{array}$ & 1.3722 & 0.637219 \\
\hline \multirow{2}{*}{$\begin{array}{l}\text { Constant-speed } \\
\text { control }\end{array}$} & $\begin{array}{l}\text { Tip transverse } \\
\text { response of beam }\end{array}$ & 1.3811 & 0.007241 \\
\hline & $\begin{array}{l}\text { Angular velocity } \\
\text { of hub }\end{array}$ & 1.3810 & 0.000000 \\
\hline
\end{tabular}

Next, we consider the active position control of a flexible hub-beam system. The desired position trajectory adopted by Cai, et al. and Zhang ${ }^{18,19}$ is used here, given by

$$
\theta= \begin{cases}\frac{2 \theta_{0}}{T^{2}} t^{2} & t \leq \frac{T}{2} \\ \frac{\theta_{0}}{2}+\frac{2 \theta_{0}}{T}\left(t-\frac{T}{2}\right)-\frac{2 \theta_{0}}{T^{2}}\left(t-\frac{T}{2}\right)^{2} & \frac{T}{2}<t \leq T . \\ \theta_{0} & t>T\end{cases}
$$

Equation (53) indicates that the system rotates with an accelerated motion from the initial condition. The angular velocity of the system reaches its maximum value at the moment $T / 2$, and then the system rotates with a decelerated motion, and the angular velocity turns zero after the moment $T$. The controller to be designed is required to drive the system to a desired position and suppress the residual vibration of beam after arrival. Assume that the end position of angular displacement in Eq. (53) is $\theta_{0}=\pi / 3=60^{\circ}$. The parameter $T=2 \mathrm{~s}$ is taken in Eq. (53). In designing the active controller, the weighting matrix and the weighting scalar in Eq. (32) are chosen as $\mathbf{Q}=\operatorname{diag}(1000,100,10,1,1,1)$, and $\bar{R}=1$. The responses of the system are given in Fig. 4. It is observed from Fig. 4 that the desired position may be achieved and the residual vibration of the beam may be suppressed. Using the OKID and ERA to identify the vibration frequency, the results are shown in rows 5 and 6 of Table 1, and they are also very close to the theoretical value.

Finally, we consider the constant-speed rotation control of the hub-beam system. It is assumed that the system rotates with an accelerated motion from the initial condition. The aim of control design is to drive the system to rotate from the zero moment and reach an angular velocity $\dot{\theta}$ at moment $T$. Then, the system is required to rotate with this constant angular velocity $\dot{\theta}$. At the same time, residual vibration of the beam should be suppressed by this controller. $\dot{\theta}=1 \mathrm{rad} / \mathrm{s}$ and $T=2 \mathrm{~s}$ here. In controller design, the weighting matrix $\mathbf{Q}$ and the weighting scalar $\bar{R}$ are the same as the above cases. The responses of the system are displayed in Fig. 5. Using the input and output data to identify the vibration frequency of the system, the results are shown in rows 7 and 8 of Table 1 . Similar to the above two cases, the identification results are almost identical to the theoretical one, and the identification precision 


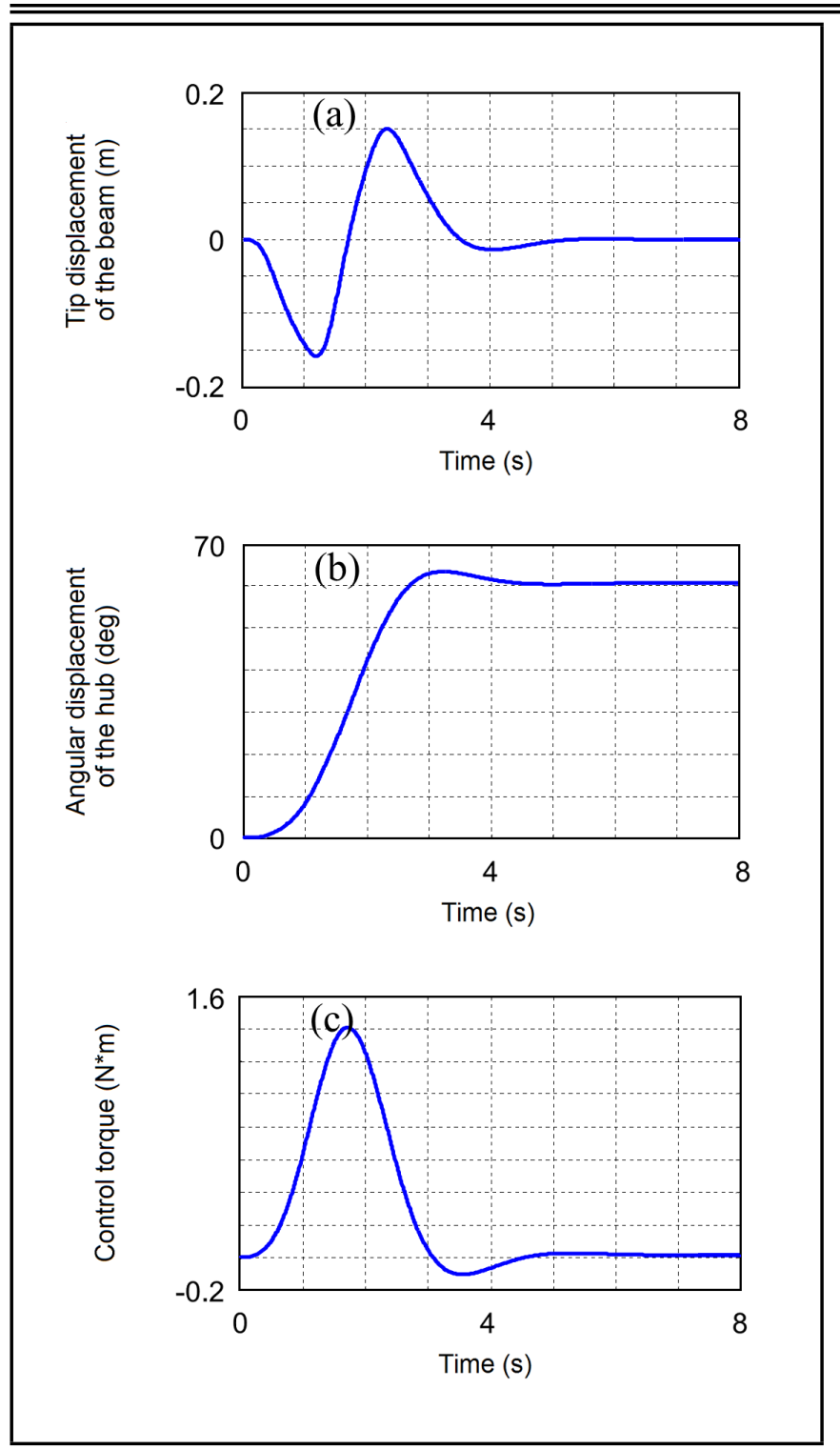

Figure 4. The responses of the system for the position control case: (a) tip displacement of the beam, (b) angular displacement of the hub, and (c) control torque.

is higher than in the above two cases, as well.

From the above simulations we can draw a conclusion that the first-order frequency of the system can be effectively identified using the OKID and ERA as long as the input and output data of the system are known. This technique may possibly be applied to the on-orbit identification of spacecraft. The onorbit identification needs the vibration response of spacecraft, and the attitude manoeuvre of spacecraft exactly provides an excitation, so it can be accomplished without adding additional space missions.

\section{CONCLUSIONS}

In this paper, active control and parameter identification are studied based on a flexible hub-beam system. The first-order approximation coupling (FOAC) model is introduced. The optimal tracking control theory is used for the active control of the system. A parameter identification technology is presented using the OKID and ERA. The research results indicate that
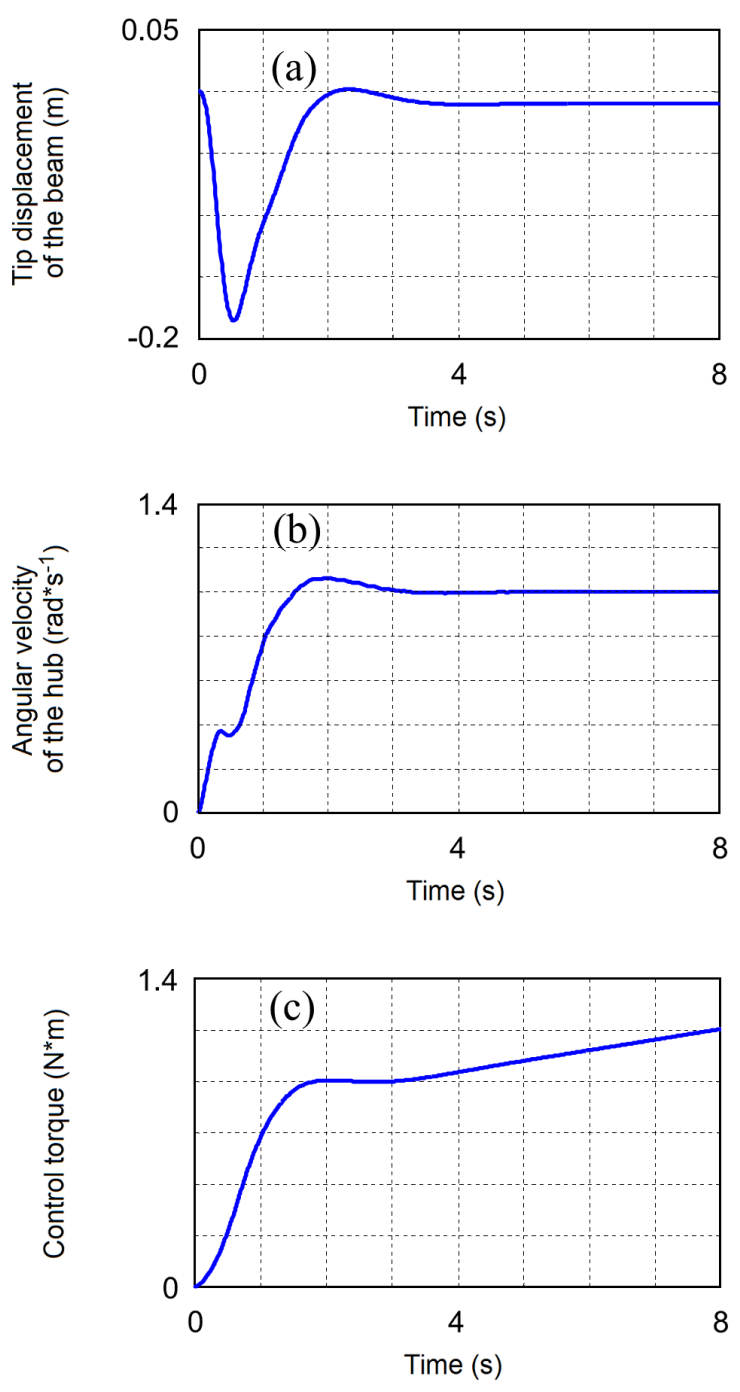

Figure 5. The responses of the system for the constant-speed case: (a) tip displacement of the beam, (b) angular velocity of the hub, and (c) control torque.

the desired position of the system may be achieved by using the controller, and the residual vibration of the beam can be suppressed, as well. The system frequency may be identified effectively by the OKID and ERA, no matter what kind of external torque is put on the hub. It is feasible and effective to obtain the system frequency using the control data.

\section{ACKNOWLEDGEMENT}

This work was supported by the Key Project (11132001) and the General Project (11272202) of Natural Science Foundation of China, the Aviation Science Foundation of China (20120157002), and the Key Scientific Project of Shanghai Municipal Education Commission (14ZZ021).

\section{REFERENCES}

${ }^{1}$ Haugse, E. D., Juengst, C. D., Salus, W. L., and Lollock, J. A. On orbit system identification, A collection of technical papers : 37th AIAA/ASME/ASCE/AHS/ASC Structures, 
Structural Dynamics, and Materials Conference, 4, 23982407, American Institute of Aeronautics and Astronautics, Washington, DC, (1996).

2 Kim, H. M., Van Horn, D. A., and Doiron, H. H. Free-decay time-domain modal identification for large space structures, Journal of Guidance, Control, and Dynamics, 17 (3), 513519, (1994).

3 Kasai, T., Komatsu, K., and Sano, M. Modal parameter identification of controlled structures, Journal of Guidance, Control, and Dynamics, 20 (1), 184-186, (1997).

4 Yamaguchi, I. and Kida, T. ETS-VI on-orbit system identification experiments, JSME International Journal, 40 (4), 623-629, (1997).

5 Adachi, S. and Yamaguchi, I. On-orbit system identification experiments on Engineering Test Satellite-VI, Control Engineering Practice, 7, 831-841, (1999).

6 Yamaguchi, I., Kida, T., and Kasai, T. Experimental demonstration of LSS system identification by eigensystem realization algorithm, Proceedings of American Control Conference, Seattle, Washington, 407-411, (1995).

7 Ishikawa, S., Yamada, K., Yamaguchi, I., Chida, Y., and Adachi, S. ETS-VI on-orbit parameter estimation by random excitation, Proceedings of Astrodynamics Symposium, Halifax, Nova Scotia, Canada, (1995).

8 Kasai, T., Komatsu, K., and Sano, M. Modal parameter identification of controlled flexible structures, AIAA Journal of Guidance, Control, and Dynamics, 20 (2), 184-186, (1997).

9 Kaouk, M., McNeill, S., Haley, S., et al. Shuttle-ISS flight7A on orbit test verification: pre and post flight analysis, Society for Experimental Mechanics, Boeing Company, (2003).

10 Kaouk, M., Haley, S., Bartkowicz, T., et al. Modal analysis and correlation of International Space Station 4A mated configuration, Society for Experimental Mechanics, Boeing Company, (2002).

11 Kim, H. M. and Bokhour, E. B. Mir structural dynamics experiment: a flight experiment development, Proceedings of the 38th AIAA SDM Conference, Kissimmee, Florida, 577585, (1997).

12 Kim, H. M. and Kaouk, M. Mir structural dynamics experiment: first phase test and data analysis, Proceedings of the 39th AIAA SDM Conference, Long Beach, CA, 204-212, (1998).
13 Kim, H. M. and Kaouk, M. Mir structural dynamics experiment: first phase test and model refinement, Proceedings of the 40th SDM Conference, St. Louis, MO, (1999).

14 Shuttle-Mir Docking Mission 5: Structural mathematical Models. STS-81, WG-03 Operations and Systems Integration Document, NASA-JSC Document \#WG-3/RSC E/NASA/000/3412-5, (1996).

15 Cai, G-P., Hong, J. Z., and Yang, S. X. Dynamic analysis of a flexible hub-beam system with tip mass, Mechanics Research Communications, 32 (2), 173-190, (2005).

16 Cai, G-P. and Lim, C. W. Active control of a flexible hubbeam system using optimal tracking control method, International Journal of Mechanical Sciences, 48 (10), 11501162, (2006).

17 Cai, G-P. and Lim, C. W. Optimal tracking control of a flexible hub-beam system with time delay, Multibody System Dynamics, 16 (4), 331-305, (2006).

18 Cai, G-P., Hong, J. Z., and Yang, S. X. Model study and active control of a rotating flexible cantilever beam, International Journal of Mechanical Sciences, 46 (6), 871-889, (2004).

19 Zhang, L. T. Study of control simulations of a flexible manipulator system, Master Thesis in Shanghai Jiaotong University, China, (2002).

${ }^{20} \mathrm{Hu}$, S. S. Automatic Control Theory, Defence Industry Press, Beijing, China, (1998).

21 Juang, J. N., Phan, M., Horta, L. G., and Longman, R. W. Identification of observer/Kalman filter Markov parameters: theory and experiments, Journal of Guidance, Control, and Dynamics, 16, 320-329, (1993).

22 Dong, X.-J., Meng, G., and Peng, J.-C. Vibration control of piezoelectric smart structures based on system identification technique: numerical simulation and experimental study, Journal of Sound and Vibration, 297 (3-5), 680-693, (2006).

23 Juang, J. N. and Phan, M. Identification and Control of Mechanical Systems, Cambridge University Press, New York, (2001).

24 Li, D.-B. Experimental Modal Analysis and Application, Science Press, Beijing, (2001).

25 Yang, H. Study of dynamic modelling theory and experiment for rigid-flexible coupling systems, $\mathrm{PhD}$ Thesis in Shanghai Jiaotong University, (2002). 